\title{
How Much Fat Loss Is Needed for Lipoatrophy to Become Clinically Evident?
}

\author{
Daniel Podzamczer, Elena Ferrer, Esteban Martínez, ${ }^{1}$ Luis del Rio, ${ }^{3}$ Joaquín Rosales, ${ }^{3}$ Jordi Curto, \\ Esteban Ribera, ${ }^{4}$ Pilar Barrufet, ${ }^{5}$ Josep M. Llibre, ${ }^{6}$ and Miquel Aranda ${ }^{7}$ for the ABCDE Study Team
}

\begin{abstract}
The objective of this study was to evaluate how much limb fat is needed to be lost for lipoatrophy to become clinically evident. Antiretroviral drug-naive patients from a randomized trial comparing stavudine or abacavir plus lamivudine and efavirenz, who had subjective assessment to detect clinically evident lipoatrophy (standardized questionnaire) and objective measurements of limb fat (dual X-ray absorptiometry) at baseline, 48 weeks, and 96 weeks were included. ROC curves were used to assess the sensitivity and specificity of several cut-off values of absolute and percent limb fat loss for diagnosing lipoatrophy. Of 54 patients included, 13 (24\%) had subjective lipoatrophy at 96 weeks. After 96 weeks, median limb fat change was $-2.3 \mathrm{~kg}$ (interquartile range: -5.2 , +0.2 ) and $0.4 \mathrm{~kg}$ (interquartile range: $-7.2,+3.4$ ) in patients with and without lipoatrophy, respectively. Median percent limb fat change was $-45.5 \%$ (interquartile range: $-78.0,+3.7$ ) and $5.5 \%$ (interquartile range: $-62.8,+95.6$ ), respectively. The cut-off values of absolute and percent limb fat loss showing the best sensitivity and specificity values were $-1.5 \mathrm{~kg}$ (sensitivity, $77 \%$; specificity, $76 \%$ ) and $-30 \%$ (sensitivity, $85 \%$; specificity, $73 \%$ ). At least $30 \%$ limb fat is needed to be lost in HIV-infected patients for lipoatrophy to become clinically evident.
\end{abstract}

\section{Introduction}

L ONG-TERM TOXICITY such as metabolic disturbances and body fat changes remains an important limitation of highly active antiretroviral therapy (HAART). ${ }^{1-3}$ The latter, known as lipodystrophy, is a complex feature, whose pathogenesis is not completely understood. ${ }^{1}$ It is a stigmatizing condition that leads to psychological and social problems, makes adherence to therapy difficult, and is sometimes associated with metabolic alterations. Lipodystrophy may present as central fat increase, known as lipohypertrophy, peripheral (face, limbs, and buttocks) fat loss, termed lipoatrophy, or both manifestations. ${ }^{1,2}$ Among other factors, such as the effect of HIV infection itself, ${ }^{4}$ protease inhibitors have been cited as participating in the development of lipohypertrophy, whereas thymidine analogs, through a mechanism of mitochondrial toxicity, seem to play an important role in lipoatrophy. 3,5
Lipodystrophy is usually defined clinically. Although the subjective definition may be easy to use in the clinical setting, it is less useful for clinical research. Several methods, such as dual energy X-ray absorptiometry (DEXA), anthropometric measures, computed tomography scanning, and others, ${ }^{6,7}$ can assess body fat objectively, but they do not directly provide the diagnosis of lipodystrophy. Although several attempts have been made to develop an objective definition, there is no clear operative definition for lipodystrophy. ${ }^{6-8}$

In two randomized clinical trials, fat changes were assessed using an arbitrary criterion of $\geq 20 \%$ peripheral fat loss measured by DEXA to define lipoatrophy., ${ }^{9,10}$ In these studies, efavirenz combined with two nucleosides was found to be associated with a greater incidence of this so-defined lipoatrophy when compared with lopinavir combined or not with two nucleosides. ${ }^{9,10}$ To understand the clinical relevance of these findings, it is important to know the relationship between $a \geq 20 \%$ peripheral fat loss and the diagnosis of clinically

\footnotetext{
${ }^{1}$ Hospital Universitari de Bellvitge, Barcelona, Spain

${ }^{2}$ Hospital Clinic, Barcelona, Spain.

${ }^{3}$ CETIR, Barcelona, Spain.

${ }^{4}$ Hospital Vall d'Hebron, Barcelona, Spain.

${ }^{5}$ Hospital de Mataró, Mataró, Spain.

${ }^{6}$ Hospital Germans Trias, Badalona, Spain

${ }^{7}$ Hospital de Terrassa, Terrassa, Spain.
} 
evident lipoatrophy. Thus, the objective of this study was to evaluate how much limb fat is needed to be lost for lipoatrophy to become clinically evident in a subset of antiretroviral drug-naive HIV-infected patients from a randomized clinical trial who had subjective assessment of lipoatrophy and objective measurements of limb fat.

\section{Patients and Methods}

The ABCDE study was a 96-week randomized clinical trial evaluating as primary end point the incidence of lipoatrophy in $237 \mathrm{HIV}$-infected naive patients initiating lamivudine/ efavirenz plus stavudine or abacavir. ${ }^{11}$ Lipoatrophy was assessed by a predefined questionnaire modified from previous publications, $^{12}$ performed at 48 and 96 weeks. Anthropometric measurements performed at the same time points according to standard techniques are described elsewhere. ${ }^{13}$ Lipoatrophy features included decreased subcutaneous fat tissue in the face, buttocks, and/or extremities. The morphological abnormality at the precise site was diagnosed when reported by the patient and confirmed by medical examination. ${ }^{11}$ In addition, after giving their informed consent, patients from six participating centers located near the DEXA center (Centro Técnico de Isótopos Radioactivos, CETIR, Barcelona, Spain) underwent DEXA scanning (Lunar DPX-L Equipment, Madison, WI) at baseline and at 48 and 96 weeks. Clinical assessment and DEXA were performed by different investigators blinded to the results of the other evaluation.
Data from patients with DEXA scan and clinical evaluation were analyzed for the present study.

Descriptive statistics of baseline characteristics were calculated overall and for each group according to clinically diagnosed lipoatrophy. A comparison of limb fat loss was performed between groups established according to their baseline total fat evaluated by DEXA or baseline weight (patients distributed into two groups, those above and below the median values of total fat and weight). Receiver operating characteristic (ROC) analysis was used to assess the sensitivity and specificity of absolute and percent limb fat loss as diagnostic criteria for defining lipoatrophy. Sensitivity and specificity were calculated for every $0.5 \mathrm{~kg}$ of absolute limb fat loss and every $5 \%$ of percent limb fat loss.

\section{Results}

In the ABCDE study, a lower proportion of patients assigned to abacavir developed clinical signs of lipoatrophy $(4.8 \%$ vs. $38.3 \% ; p<0.001)$.

Fifty-four patients had a clinical evaluation and DEXA scan performed at the three time points: $13(24 \%)$ were clinically diagnosed with lipoatrophy (LA) and 41 (76\%) were not (no-LA). As mentioned in the original publication of the ABCDE study, ${ }^{11}$ within this subgroup of patients, both arms presented baseline characteristics similar to those of the total study population except for serum cholesterol concentration (data not shown). The groups were well balanced for baseline

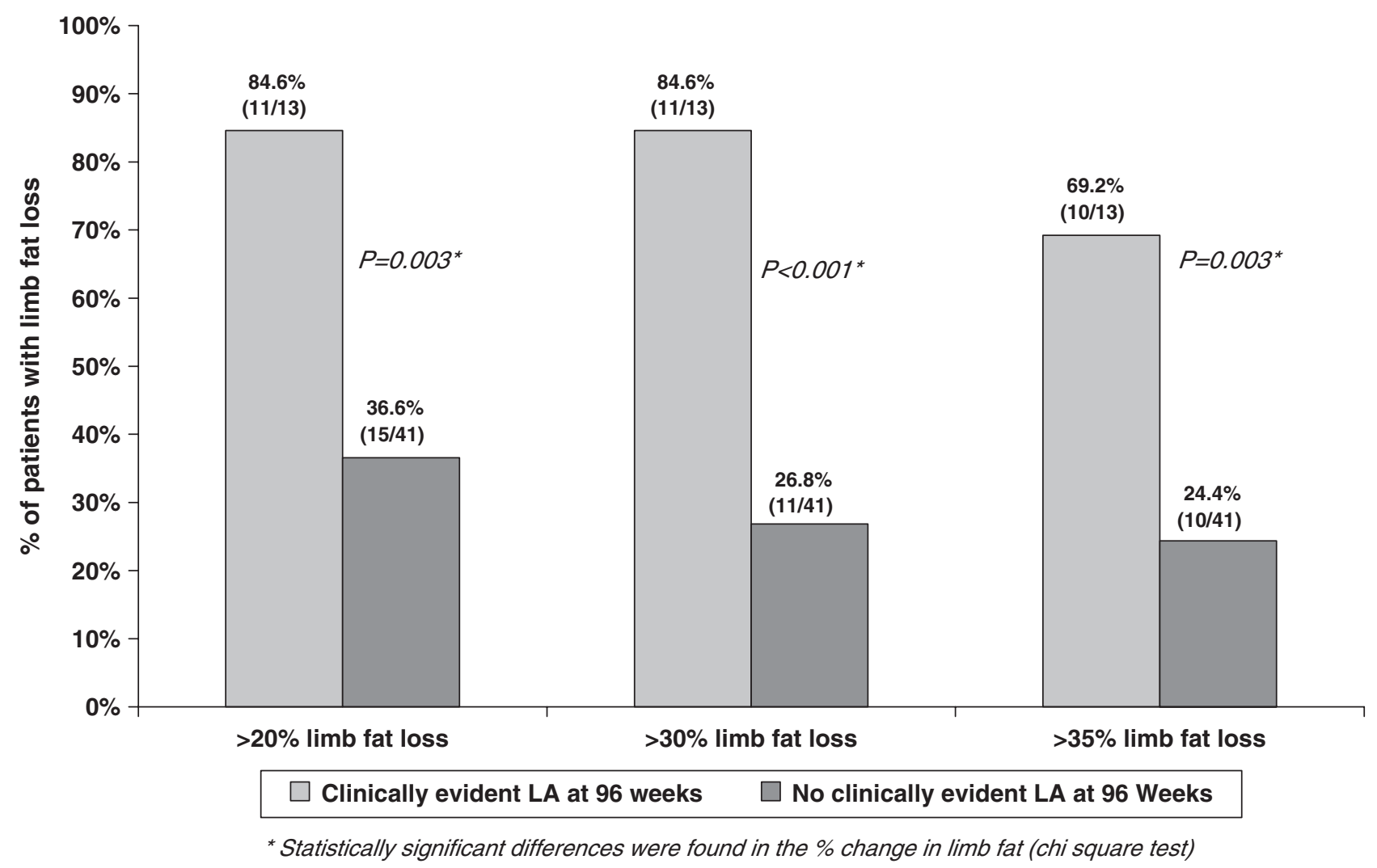

FIG. 1. Sensitivity and specificity of three different proportions of limb fat loss on DEXA (20\%, 30\%, and 35\%) for the diagnosis of clinically evident lipoatrophy (LA). 
characteristics, with $77.8 \%$ men and $88.9 \%$ white. HIV risk groups were distributed as follows: $11.1 \%$ former drug users, $33.3 \%$ homosexuals, $46.3 \%$ heterosexuals, and $9.3 \%$ others. Among the total, 16.7\% had prior AIDS, median CD4 was 196 cells / $\mu \mathrm{l}$, and median viral load was 99,265 copies $/ \mathrm{ml}$. Patients received lamivudine/efavirenz plus stavudine in $53.7 \%$, and lamivudine/efavirenz plus abacavir in $46.3 \%$. Median weight was $65 \mathrm{~kg}$, and median limb fat on DEXA was $5.7 \mathrm{~kg}$. The only variable showing a significant difference between groups was age: median of 39 years in the no-LA group and 50 years in the LA group $(p=0.036)$.

After 48 weeks, median absolute limb fat was $5.8 \mathrm{~kg}$ (range $4.2 ; 7.6)$ in the no-LA group and $3.9 \mathrm{~kg}(3.1 ; 5.1)$ in the LA group ( $p=0.073)$, median limb fat loss was $-0.2 \mathrm{~kg}(-6.0 ; 3.4)$ in no-LA and $-1.9 \mathrm{~kg}(-3.9 ; 3.8)$ in LA $(p=0.199)$, and median percent limb fat change was $-2.4 \%(-63.8 ; 107.6)$ in no-LA and $-34.7 \%(-77.3 ; 89.1)$ in LA $(p=0.108)$. After 96 weeks, the median absolute limb fat was $6.2 \mathrm{~kg}(3.0 ; 8.1)$ in no-LA and $3.0 \mathrm{~kg}(1.6 ; 4.0)$ in LA $(p=0.002)$, median limb fat loss was $0.4 \mathrm{~kg}(-7.2 ; 3.4)$ in no-LA and $-2.3 \mathrm{~kg}(-5.2 ; 0.2)$ in LA $(p=0.002)$, and median percent limb fat change was $5.5 \%$ $(-62.8 ; 95.6)$ in no-LA and $-45.5 \%(-78.1 ; 3.7)$ in LA $(p<0.001)$.

The proportion of limb fat loss was independent of total fat assessed by DEXA at baseline $(\leq 15 \mathrm{~kg}$ [median baseline total fat of the 54 patients] vs. $\geq 15 \mathrm{~kg}$ ). In patients with baseline total fat $\leq 15 \mathrm{~kg}$, the percent limb fat change at 96 weeks was $7.5 \%$ $(-49.3 ; 95.6)$ in the no-LA group and $-42.9 \%(-78.1 ; 3.7)$ in the LA group $(p=0.019)$, whereas in patients with baseline total fat $>15 \mathrm{~kg}$ the percent limb fat change was $3.8 \%(-62.8 ; 40.9)$ in no-LA and $-48.4 \%(-57.9 ;-32.8)$ in LA $(p=0.006)$. Similarly, the proportion of limb fat loss was independent of baseline weight ( $\leq 965 \mathrm{~kg}$ [median baseline weight of the 54 patients] vs. $>65 \mathrm{~kg}$ ). In patients with a baseline weight $\leq 965 \mathrm{~kg}$, the percent limb fat change at 96 weeks was $0.6 \%(-49.3 ; 95.6)$ in the no-LA group and $-47.0 \%(-78.1 ; 3.7)$ in the LA group $(p=0.006)$, and in patients with baseline weight $>65 \mathrm{~kg}$, the percent limb fat change was $7.5 \%(-62.8 ; 91.5)$ in no-LA and $-40.0 \%(-57.9 ;-15.2)$ in LA $(p=0.040)$.

The sensitivity and specificity of three different percentages of limb fat loss evaluated by DEXA $(20 \%, 30 \%$, and $35 \%)$ for diagnosing clinically evident LA are shown in Fig. 1. ROC curves showing the sensitivity and specificity of absolute and percent limb fat changes for diagnosing clinically evident LA are presented in Fig. 2. Thirty percent was the best cut-off for percent limb fat loss (sensitivity, 85\%; specificity, 73\%), whereas $1.5 \mathrm{~kg}$ was the best cut-off in terms of absolute changes in limb fat loss (sensitivity, 77\%; specificity, 76\%) (sensitivity and specificity for other cut-offs are not shown).

\section{Discussion}

To our knowledge, this is the first study that attempts in a prospective way to correlate the percentage of peripheral fat loss evaluated by DEXA scanning with the presence of clinically evident LA.

If the $\geq 20 \%$ fat loss cut-off used to define LA in the ACTG5142 and M03-613 studies $^{9,10}$ had been used in our study, more than one-third (36.6\%; Fig. 1) of patients without clinically evident LA would have been "erroneously" diagnosed as having LA. On the other hand, if the cut-off had been $\geq 35 \%$, a reduction in false-positive LA to $24.4 \%$ would occur,

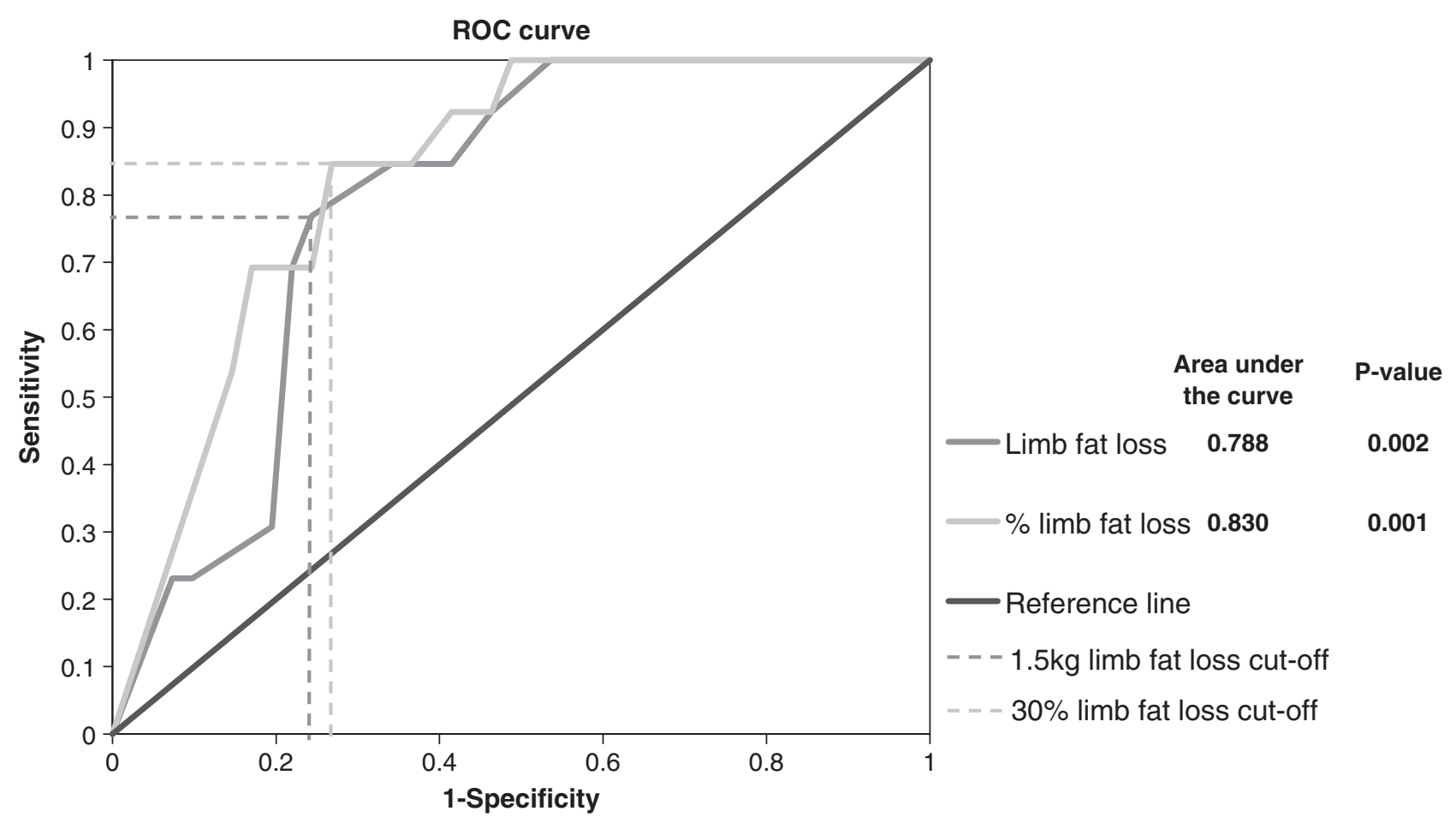

FIG. 2. ROC curves show the sensitivity and specificity of absolute and percent limb fat changes for diagnosing clinically evident lipoatrophy. 
but sensitivity (patients with a clinical diagnosis of LA identified as LA) would decrease from $84.6 \%$ to $69.2 \%$. Thus, based on the ROC curves, $30 \%$ was the best cut-off in terms of sensitivity and specificity for the diagnosis of clinically evident LA. However, we should recognize that, approximately one-quarter of patients without clinical lipoatrophy would be diagnosed of lipoatrophy using this 30\% cut-off.

These data are in agreement with an analysis of data from ACTG 5142, in which investigators realized that most patients with $\geq 20 \%$ peripheral fat loss did not present evident lipoatrophy. ${ }^{14}$

These findings do not try to minimize the potential relevance of losing $20 \%$ of limb fat, but it should be noted that this amount of fat loss is not evident to the observer in most cases. Actually, median limb fat loss in our patients was $45 \%$, far from the $20 \%$ used to define lipoatrophy in the aforementioned clinical trials. Another consideration is that a loss of $20 \%$ of peripheral fat may herald an increasing loss in the next months or years. This possibility should be examined in large prospective surveys that include clinical evaluation and DEXA scanning. If this is confirmed, we hypothesize that the preclinical finding of $20 \%$ or less fat loss might be a useful signal to switch antiretroviral regimens to prevent the development of clinically evident lipoatrophy; that is, to act before crossing the $20 \%$ line.

Although thymidine analogs, the nucleoside analogs most often related with lipoatrophy, have been relegated as alternative treatment options for antiretroviral drug-naive patients, ${ }^{15}$ the long-term probability of developing this complication with use of the less toxic nonthymidine analogs is unknown. In addition, a role of other antiretroviral compounds in lipoatrophy through different mechanisms cannot be ruled out. ${ }^{16}$

This study has the limitation of a small sample size; thus, our findings should be taken with caution and confirmed in larger series. In addition, although we have observed that the high percentage of peripheral fat loss is independent of baseline total fat or weight, we do not know whether our data apply similarly to individuals of both sexes, or to different age groups or races.

In conclusion, $>20 \%$ peripheral fat loss is not clinically evident in most patients. Hence, and despite its limitations, $>30 \%$ might be a better cut-off to objectively define lipoatrophy in HIV-infected patients under antiretroviral therapy. If this cut-off value is confirmed in further studies, it could be of use for clinical care and research purposes.

\section{Acknowledgments}

We acknowledge Isaac Ruiz, M.S.C., for his help in statistical analysis. This work was supported in part by the Fundación para la Investigación y la Prevención del SIDA en España (FIPSE, Foundation for AIDS Research and Prevention; grant 36274/02) and Red de Investigación en SIDA (RIS, AIDS Research Network; Redg173), and was presented at the 15th Conference on Retroviruses and Opportunistic Infections (Boston, MA), February 3-6, 2008 (abstract 941).

\section{Author Disclosure Statement}

D. Podzamczer, E. Ferrer, E. Martínez, E. Ribera, J.M. Llibre, and P. Barrufet have received research grants and/or an honorarium for advisories or lectures from Abbott, Bristol-
Myers Squibb, and other pharmaceutical manufacturers of antiretroviral agents. No conflict of interest was reported by the other authors.

\section{References}

1. Carr A: HIV lipodystrophy: Risk factors, pathogenesis, diagnosis and management. AIDS 2003;17(Suppl 1):S141S148.

2. Hadigan C, Meigs JB, Corcoran C, et al.: Metabolic abnormalities and cardiovascular disease risk factors in adults with human immunodeficiency virus infection and lipodystrophy. Clin Infect Dis 2001;32:130-139.

3. Carr A, Samaras K, Chrisholm DJ, et al.: Pathogenesis of HIV-1-protease inhibitor-associated peripheral lipodystrophy, hyperlipidaemia, and insulin resistance. Lancet 1998; 351:1881-1883.

4. Bacchetti P, Gripshover B, Grunfeld C, Heymsfield S, McCreath H, Osmond D, Saag M, Scherzer R, Shlipak M, Tien P; Study of Fat Redistribution and Metabolic Change in HIV Infection (FRAM): Fat distribution in men with HIV infection. JAIDS 2005;40:121-131.

5. Brinkman K, Smeitink JA, Romijn JA, and Reiss P: Mitochondrial toxicity induced by nucleoside-analogue reversetranscriptase inhibitors is a key factor in the pathogenesis of antiretroviral-therapy-related lipodystrophy. Lancet 1999; 354:1112-1115.

6. Schwenk A: Methods of assessing body shape and composition in HIV-associated lipodystrophy. Curr Opin Infect Dis 2002;15:9-16.

7. Pritchard JE, Nowson CA, Staruss BJ, et al.: Evaluation of dual energy X-ray absorptiometry as a method of measurement of body fat. Eur J Clin Nutr 1993;47:216-228.

8. Carr A, Emery S, Law M, et al.: An objective case definition of lipodistrophy in HIV-infected adults: A case-control study. Lancet 2003;361:726-735.

9. Haubrich R, Riddler S, DiRienzo D, et al.: Metabolic outcomes of ACTG5142: A prospective, randomized, phase III trial of NRTI-, PI-, and NNRTI-sparing regimens for initial treatment of HIV-1 infection. Presented at the 14th Conference on Retroviruses and Opportunistic Infections, February 25-28, 2007. Los Angeles, CA, Abstract 38.

10. Cameron DW, da Silva B, Arribas J, et al.: A 96-week comparison of lopinavir-ritonavir combination therapy followed by lopinavir-ritonavir monotherapy versus efavirenz combination therapy. J Infect Dis 2008;198:234-240.

11. Podzamczer D, Ferrer E, Sanchez P, et al.: Less lipoatrophy and better lipid profile with abacavir as compared to stavudine: 96-week results of a randomized study. JAIDS 2007; 44:139-147.

12. Lichtenstein KA, Ward DJ, Moorman AC, et al.: Clinical assessment of HIV-associated lipodystrophy in an ambulatory population. AIDS 2001;15:1389-1398.

13. Lohman TG, Roche AF, Martorell R: Anthropometric Standardization Reference Manual. Champaign, IL: Human Kinetics Books; 1988.

14. Haubrich R, Riddler S, DiRienzo G, et al.; ACTG 5142 Study Team. Clinical associations of extremity fat loss: ACTG 5142, a prospective, randomized, phase III Trial of NRTI-, PI-, and NNRTI-sparing regimens for ART of naive, HIV-1-infected subjects. Presented at the 15th Conference on Retroviruses and Opportunistic Infections; February 3-6, 2008; Boston, MA. Abstract 935.

15. DHHS Panel on Antiretroviral Guidelines for Adults and Adolescents: Guidelines for the Use of Antiretroviral Agents 
in HIV-1-Infected Adults and Adolescents, January 29, 2008. Available at: http://www.AIDSinfo.nih.gov/ContentFiles/ AdultandAdolescentGL.pdf.

16. El Hadri K, Glorian M, Monsempes $\mathrm{C}$, et al:: In vitro suppression of the lipogenic pathway by the nonnucleoside reverse transcriptase inhibitor efavirenz in 3T3 and human preadipocytes or adipocytes. J Biol Chem 2004;279:1513015141.
Address reprint requests to: Daniel Podzamczer Infectious Disease Service Hospital Universitari de Bellvitge c/Feixa Llarga s/n. L'Hospitalet 08907 Barcelona, Spain

E-mail: dpodzamczer@csub.scs.es 